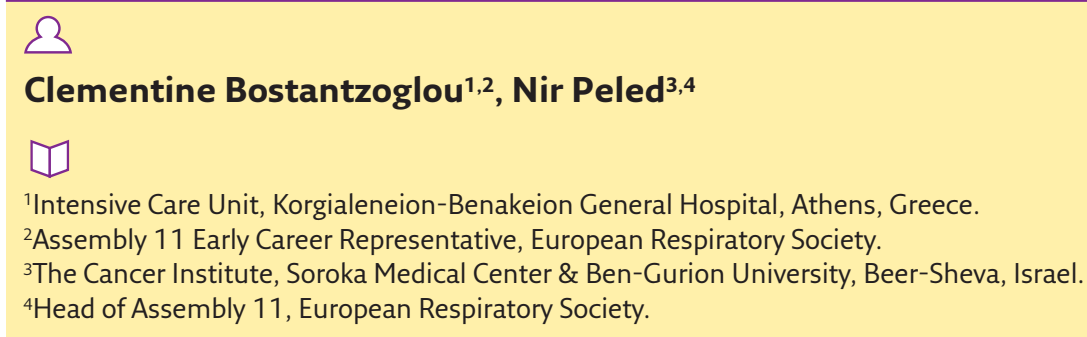

${ }^{1}$ Intensive Care Unit, Korgialeneion-Benakeion General Hospital, Athens, Greece.

${ }^{2}$ Assembly 11 Early Career Representative, European Respiratory Society.

${ }^{4} \mathrm{Head}$ of Assembly 11, European Respiratory Society.

\title{
Assembly 11: thoracic oncology
}

\section{Meet the Assemblies}

Assembly 11 is the European Respiratory Society Assembly that focuses on lung cancer, mesothelioma, mediastinal tumours and lung metastases. It is an interdisciplinary and multi-professional group whose members' expertise extends from prevention and screening, to diagnosis and staging, and from systematic treatment with chemotherapy and targeted agents to follow-up and supportive care.

Lung cancer is one of the most common malignancies and the leading cause of death from cancer worldwide. According to the World Health Organization, the estimated number of new cases was 1.8 million in 2012. In the USA alone, the American Cancer Society estimates that in 2018 there will be more than 234,000 new cases. Lung cancer is not only one of the most common malignancies but it is also one of the deadliest, being responsible for nearly one out of five (1.59 million deaths, $19.4 \%$ of the total) deaths from cancer worldwide [1, 2].

Lung cancer has long been in the spotlight of scientific research as it affects the lives of millions of people.

Disease is lacking early symptoms, which results, for the majority of new cases, in diagnosis at advanced stages where prognosis is poor. The issue of screening for lung cancer has been addressed in numerous studies. The National Lung Screening Trial is the largest study published to date. The study showed that $20 \%$ more patients were diagnosed with low-dose computed tomography and, more importantly, 50\% more patients were diagnosed at stage I-II where the disease has high cure rates (stage I-II: 158 versus 70 participants in the computed tomography screening versus the chest radiograph screening groups; and stage IIB to IV: 120 versus 111, respectively). The study did not manage to demonstrate differences in overall mortality; however, it was only a 3-year trial so we can expect more from more long-term trials [3].

Treatment for patients with advanced nonsmall cell lung cancer has historically consisted of systemic cytotoxic chemotherapy and platinumbased chemotherapy and remains the most efficacious option. However, over the past decade the improved understanding of molecular pathways that drive oncogenesis has led to the development of agents targeting specific molecular pathways. The identification of mutations in the epidermal growth factor receptor (EGFR), and rearrangements of the anaplastic lymphoma kinase $(A L K)$ gene and ROS1 gene has resulted in the development of specific molecular treatments for patients carrying these mutations. The use of such targeted therapies has improved median overall survival in the group of non-small cell lung cancer patients whose tumours harbour these alterations [4].

Furthermore, recent studies have elucidated the multiple ways in which lung cancer cells evade the immune system, and novel agents targeting this activity have emerged. Checkpoint inhibitors targeting cytotoxic T-lymphocyte-associated
Cite as: Bostantzoglou C, Peled N. Assembly 11: thoracic oncology. Breathe 2018; 14: 163-164. 
antigen- 4 and the programmed death- 1 pathway have shown promising results, along with manageable toxicities in previously heavily treated lung cancer patients. Recently, a combination therapy of immunochemo therapy has been approved by the US Food and Drug Administration and may bring a new era to our patients [5].

Based on the finding that in the blood of patients with lung cancer cell-free ctDNA and/or circulating tumour cells are often present, a number of bloodbased tests or so-called "liquid" biopsies have emerged in an attempt to genotype the disease in a less invasive yet efficacious manner.

Pulmonogists have a cardinal role throughout the management of lung cancer patients. They are responsible for the promotion and application of smoking cessation programmes. They provide their expertise as part of multidisciplinary teams in accredited medical centres, organising lung cancer screening activities. Respiratory physicians have an irreplaceable role in the primary evaluation of suspected pulmonary lesions, organising an optimal diagnostic approach and establishing diagnosis through interventional techniques. Furthermore, they participate in multidisciplinary teams organising treatment strategies and also are actively involved in the management of respiratory comorbidities and delivery of supportive care for lung cancer patients.

The aspiration of Assembly 11 is to further reinforce the role of respiratory physicians in thoracic oncology. Educational activities organised by the Assembly aim to continuously train and update members regarding prevention, screening and early detection of lung cancer, delivering precise diagnosis and optimum personalised care for early or advanced stages of disease. There is cooperation and networking with other Assemblies, such as Basic and Translational Sciences (Assembly 3) and Thoracic Surgery and Transplantation (Assembly 8), as well as other societies such as the European Society of Radiology and the European Society of Thoracic Surgeons. At the 2017 European Respiratory Society International Congress we were responsible for several activities including a grand round session, two postgraduate courses, two meet the expert sessions and a case-based discussion activity.

We would like to make a call for young, engaged members to join us, in an effort to create a network of clinicians and researchers interested in thoracic oncology. We aim to increase the opportunity to engage people in the educational and scientific activities of the Assembly.

\section{Conflict of interest}

N. Peled reports grants and personal fees (advisor and honorarium) from AstraZeneca, Boehringer Ingelheim, BMS, Lilly, MSD, Novartis, Pfizer, Roche, NovellusDx, FMI and Gaurdant360, outside the submitted work.

\section{References}

1. International Agency for Research on Cancer. GLOBOCAN 2012: estimated cancer incidence, mortality and prevalence worldwide in 2012. http://globocan.iarc.fr/Pages/fact_ sheets_cancer.aspx Date last updated: 2012. Date last accessed: April 18, 2018

2. American Cancer Society. Key statistics for lung cancer. www. cancer.org/cancer/non-small-cell-lung-cancer/about/ key-statistics.html Date last updated: January 4, 2018. Date last accessed: April 18, 2018

3. National Lung Screening Trial Research Team, Church TR Black WC. Results of initial low-dose computed tomographic screening for lung cancer. N Engl J Med 2013; 368: 1980-1991.

4. Travis WD, Brambilla E, Nicholson AG, et al. The 2015 World Health Organization classification of lung tumors: impact of genetic, clinical and radiologic advances since the 2004 classification. J Thorac Oncol 2015; 10: 1243-1260.

5. Rizvi NA, Mazières J, Planchard D, et al. Activity and safety of nivolumab, an anti-PD-1 immune checkpoint inhibitor, for patients with advanced, refractory squamous non-small-cell lung cancer (CheckMate 063): a phase 2, single-arm trial. Lancet Oncol 2015; 16: 257-265. 